\title{
Differentiation induced by physiological and pharmaco- logical stimuli leads to increased antigenicity of human neuroblastoma cells
}

\author{
Lena-Maria Carlson ${ }^{1}$, Sven Påhlman ${ }^{2}$, Anna De Geer ${ }^{1}$, Per Kogner ${ }^{3}$, Jelena Levitskaya ${ }^{1,4}$ \\ ${ }^{1}$ Immune and Gene Therapy Unit, Cancer Centrum Karolinska, Karolinska Institutet, Karolinska Hospital, KS-ringen, R8:01, \\ S-17176 Stockholm, Sweden; ${ }^{2}$ Division of Molecular Medicine, Department of Laboratory Medicine, Lund University, S-20502 \\ Malmö, Sweden; ${ }^{3}$ Childhood Cancer Research Unit, Department of Woman and Child Health, Karolinska Institutet, S-17176 \\ Stockholm, Sweden
}

Sympathetic neuronal differentiation is associated with favorable prognosis of neuroblastoma (NB), the most common extra-cranial solid tumor of early childhood. Differentiation agents have proved useful in clinical protocols of NB treatment, but using them as a sole treatment is not sufficient to induce tumor elimination in patients. Therefore, complementary approaches, such as immunotherapy, are warranted. Here we demonstrate that differentiation of NB cell lines and ex vivo isolated tumor cells in response to physiological or pharmacological stimuli is associated with acquisition of increased antigenicity. This manifests as increased expression of surface major histocompatibility class I complexes and ICAM-1 molecules and translates into increased sensitivity of NB cells to lysis by cytotoxic T lymphocytes (CTLs) and natural killer (NK) cells. The latter is paralleled by enhanced ability of differentiated cells to form immune conjugates and bind increased amounts of granzyme B to the cell surface. We demonstrate, for the first time, that, regardless of the stimulus applied, the differentiation state in NBs is associated with increased tumor antigenicity that enables more efficient elimination of tumor cells by cytotoxic lymphocytes and paves the way for combined application of differentiation-inducing agents and immunotherapy as an auxiliary approach in NB patients.

Keywords: neuroblastoma, differentiation, antigenicity, cytotoxic lymphocytes, MHC

Cell Research (2008) 18:398-411. doi: 10.1038/cr.2008.27; published online 12 February 2008

\section{Introduction}

Differentiation is a cell lineage-specific program of gene expression and gene silencing, which results in generation of specialized cells (reviewed in [1]). After reaching the stage of terminal differentiation, normal cells irreversibly lose their proliferative capacity. It is believed that cancer development and progression often is associated with derangement of the microenvironment, i.e. in-

Correspondence: Jelena Levitskaya

Tel: +46-8-51776876; Fax: +46-8-309195

E-mail: Elena.Levitskaya@ki.se

${ }^{4}$ Current address: W. Harry Feinstone Department of Molecular Microbiology and Immunology, Johns Hopkins Bloomberg School of Public Health, 615 N. Wolfe Street Baltimore, MD 21205, USA

E-mail: jelevits@jhsph.edu

Received 2 August 2007; revised 25 September 2007; accepted 8 October 2007; published online 12 February 2008 activation of a normal tissue-specific regulatory network, which in turn triggers de-differentiation of tumor cells (reviewed in [2]).

Neuroblastoma (NB), the most common extra-cranial solid tumor of early childhood, originates from immature cells of the sympathetic nervous system. Multiple in vitro and in vivo studies (reviewed in [3]) have demonstrated that the degree of sympathetic neuronal differentiation affects the outcome of the disease. Differentiationinducing agents (DA), such as retinoic acid derivatives, retinoids, have been included into the standard protocols of NB treatment and proved to be effective in controlling tumor growth (reviewed in [4]). Different molecular mechanisms responsible for retinoid-mediated suppression of NB progression are known, including induction of growth arrest and/or apoptosis in the tumor cells. In addition, we have previously observed that retinoids can modulate the major histocompatibility (MHC) class I 
presentation pathway and render NBs sensitive to cytotoxic T lymphocytes (CTLs) [5]. This suggests that NB cells differentiated by retinoids may be more efficiently targeted by the immune system in NB patients. However, retinoids are known as pleiotropic agents; therefore, it is not clear whether acquisition of increased immunogenicity is an intrinsic feature of the differentiated NB phenotype or a reflection of other effects of retinoids, unrelated to differentiation.

It is known that the presence of tumor infiltrating lymphocytes (TILs) in the tumor mass correlates with favorable prognosis in NB patients [6]. It is also well documented that low expression levels of marker genes of neuronal differentiation correlate with highly malignant tumor behavior $[7,8]$. On the basis of these observations we speculated that differentiation of NBs is accompanied by changes in the tumor immune phenotype and that the failure of NB cells to maintain a differentiated phenotype may result, among other effects, in low tumor susceptibility to effector functions of the immune system. This raised the question whether differentiation induced by other DAs, distinct from retinoic acid, will be accompanied by changes in the immunogenicity of NB cells.

Different agents are capable of triggering differentiation of NB cells in vitro [3]; these include non-specific activators, such as 12-O-tetradecanoylphorbol 13-acetate (TPA), a combination of epidermal growth factor (EGF) with basic fibroblast growth factor (FGF), as well as nerve growth factor (NGF), a natural ligand for the highaffinity surface receptor trkA. We have utilized these agents to induce differentiation in in vitro propagated NB cell lines as well as in tumor cells freshly isolated from NB patients during the course of surgical intervention. We demonstrate that acquisition of a differentiated phenotype induced in NB cells by these agents is accompanied by up-regulation of MHC class I complexes and the adhesion molecule ICAM-1 at the tumor cell surface, improved binding of the apoptosis-inducing protein granzyme B, as well as enhanced lysis of NB cells by the CD8+ MHC-class I-restricted CTLs and natural killer (NK) lines.

\section{Results}

$N B$ cell lines undergo differentiation in response to neural stem cell medium (NSCM) and TPA and up-regulate MHC class I

We observed that culturing in fetal calf serum (FCS)free DMEM:F12 medium containing B-27, EGF, FGF and LIF, originally described as growth medium for propagation of neural stem cells [9] and herein referred to as NSCM, induces differentiation-associated changes such as neurite outgrowth (Figure 1A), reduction in cell proliferation (Figure 1B) and enhanced expression of the neuronal marker GAP43 (Figure 1C) in SH-SY5Y and SK-N-SH cell lines. These changes were similar to those triggered in the NB lines by TPA (Figure 1A-C), a well-documented inducer of NB cell differentiation [3]. Interestingly, NSCM significantly reduced proliferation of FL-2 cells but failed to induce GAP43 expression and the cell morphology characteristic for NB differentiation (data not shown), whereas TPA efficiently induced differentiation in the FL-2 cell line (Figure 1). We also observed a decrease in GAP43 expression in TPA-differentiated FL-2 cells, which is in agreement with previously published findings on down-regulation of GAP43 expression in Schwann cells in the premyelinating stage of differentiation (Figure 1C) [10].

The level of expression and composition of the MHC class I complexes are the key parameters defining the recognition and lysis of tumor cells by the CD8+ CTLs [11]. Therefore, we investigated whether or not MHC class I molecules are affected in NB lines undergoing differentiation. Staining of TPA- or NSCM-treated NB lines with an antibody recognizing assembled MHC class I complexes (clone W6/32) revealed increased levels of $\mathrm{MHC}$ at the surface of all differentiated NB lines tested, whereas staining with antibodies specific for individual HLA alleles demonstrated heterogeneous patterns, ranging from the absence of modulation of HLA expression, i.e. HLA-A11 in response to TPA treatment, to different degrees of up-regulation, e.g. HLA-A2 expression in response to TPA and HLA-A11 expression in response to NSCM (Figure 2A). In accordance with the increase of surface MHC class I, the total pool of class I heavy chain was also augmented as monitored by western blot in total cell lysates (Figure 2B). Since the W6/32 antibody recognizes both classical HLA alleles of $\mathrm{A}, \mathrm{B}$ and $\mathrm{C}$ loci and non-classical HLAs, such as HLA-E and HLA-G, we investigated the expression of the latter molecules in the course of NB differentiation. Whereas HLA-G expression was not detected by western blot either prior to or after differentiation of NB lines (Figure 2B), HLA-E expression was detected in two out of three NB lines tested (Figure 2B); however, the levels of HLA-E did not increase in response to DAs. Furthermore, we have observed down-regulation of HLA-E expression in total lysates of SK-N-SH propagated in NSCM, which was paralleled by a decrease of total heavy chain levels, suggesting that HLA-E contributes to the total pool of MHC class I heavy chain in this cell line. Currently, there is no commercially available HLA-E-specific antibody for flow cytometry not cross-reacting with other HLA class I alleles and, therefore, surface levels of HLA-E in SK- 
A
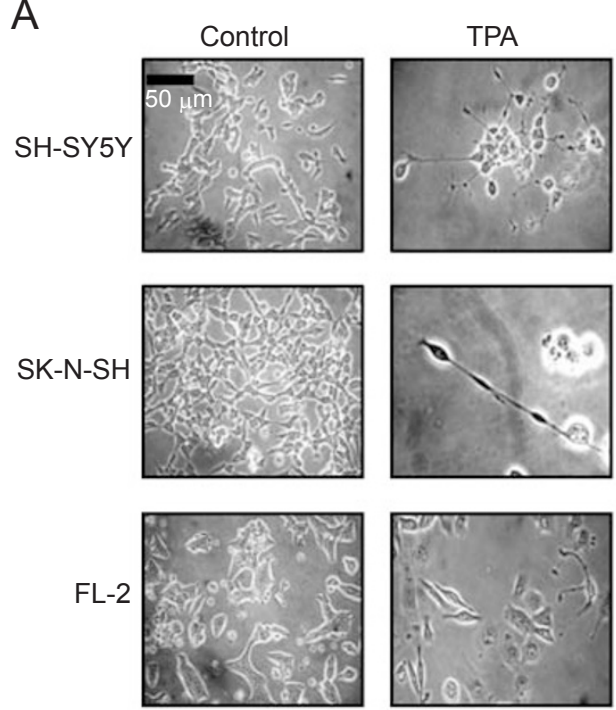

C

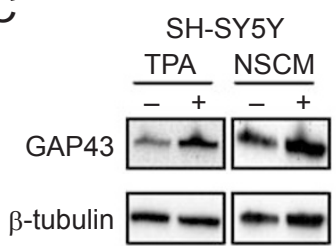

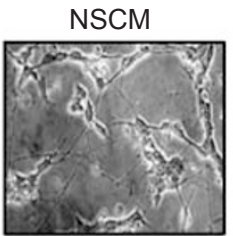
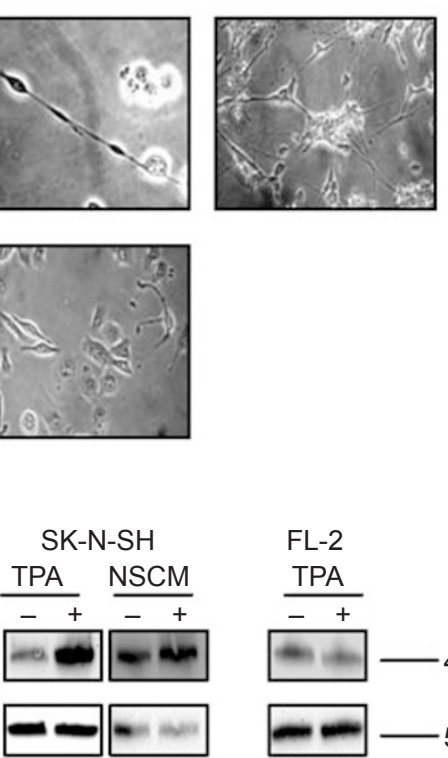

B
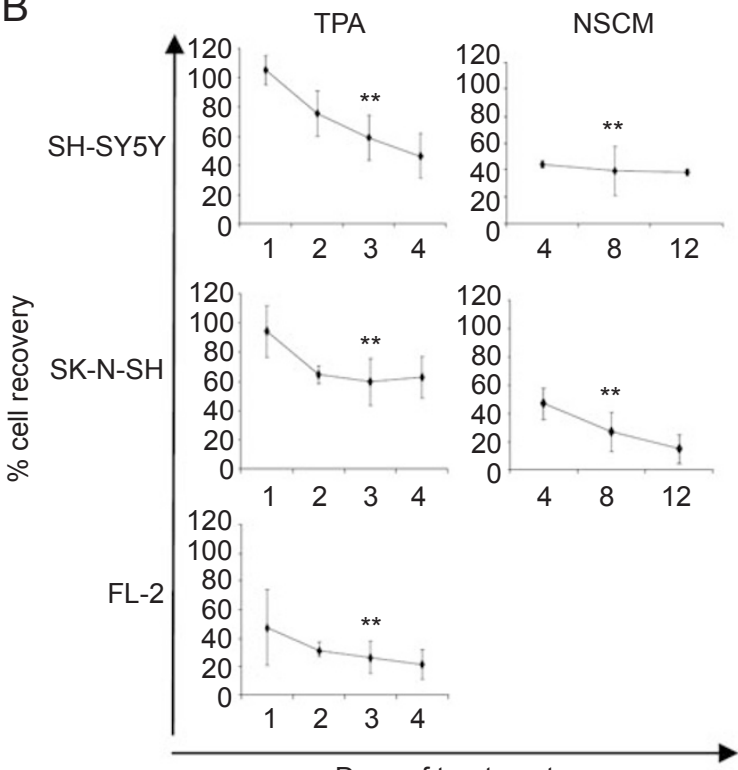

Days of treatment

Figure 1 NB cell lines undergo differentiation in response to TPA and NSCM treatment. (A) Morphological changes in NB cell lines induced by TPA on day 3 of treatment or by NSCM on day 8 of treatment were monitored using a phase-contrast microscope and digital images were acquired by a charge-coupled device. Bar $=50 \mu \mathrm{m}$. (B) Numbers of trypan-blue negative NB cells in cultures either treated with the indicated DA or left untreated were calculated at the indicated days of treatment. Cell recovery in DA-exposed cultures was calculated relative to that observed in control cultures and expressed in percent. Data from three or more experiments are presented for each time point as mean \pm SD. The value ${ }^{* *}(P<0.01)$ demonstrates significant differences obtained on day 3 or day 8. (C) Expression of GAP43 in total cell lysates of NB lines either treated with DA $(+)$ or left untreated $(-)$ was monitored by western blot. Membranes were subsequently stripped and re-probed for the expression of $\beta$-tubulin (control of sample loading). The corresponding molecular weights are indicated in $\mathrm{kDa}$.

$\mathrm{N}-\mathrm{SH}$ remain to be further determined. Thus, at least two different non-specific DAs were capable of inducing MHC class I in NB cell lines.

$N G F$ induces MHC class I in trkA-expressing NB in the course of differentiation

Expression of ectopic trkA receptor in established NB cell lines derived from highly malignant tumors leads to the restoration of their ability to differentiate in response to NGF, the natural ligand for the receptor [12-14]. Using this model we investigated whether the specific natural signal triggering differentiation in NBs influences the immunogenicity of the tumor. In accordance with previously published observations [12], the trkA-transfected (6.2 trkA), but not vector-transfected (9.1 vector) SHSY5Y cell line differentiated in response to NGF treat- ment, as demonstrated here by characteristic changes in cell morphology (Figure 3A), cell recovery (Figure 3B) and expression of the GAP43 protein (Figure 3C). The basal levels of GAP43 expression in 9.1 vector and 6.2 trkA-transfected cells were different, a phenomenon that probably reflects the artificial selection of certain subclones from original highly heterogeneous NB cultures in vitro. These changes were paralleled by an increase in total surface MHC class I complexes and HLA-A11 levels only in trkA-expressing, but not in control cells (Figure 3D). In accordance, an increase in the total pool of class I heavy chain was observed in lysates of 6.2 trkA cells, whereas neither HLA-E nor HLA-G was detected prior to or after NGF-induced differentiation (Figure 3D). These data demonstrate that specific NGF:trkA interaction results in up-regulation of MHC class I in NB cells. 
A

SH-SY5Y

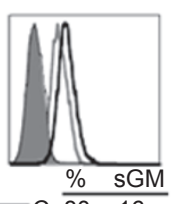

C $80 \quad 16$

- $96 \quad 27$
SK-N-SH

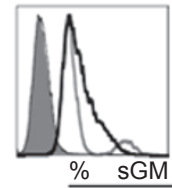

C $\overline{9130}$

T $95 \quad 39$

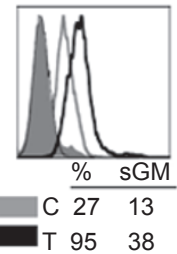

FL-2

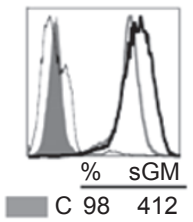

NSCM

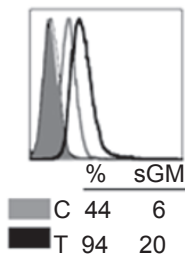

HLA ABC

SH-SY5Y
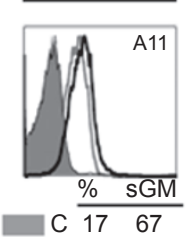

T $34 \quad 78$

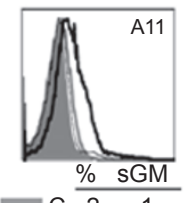

T $20 \quad 10$
SK-N-SH
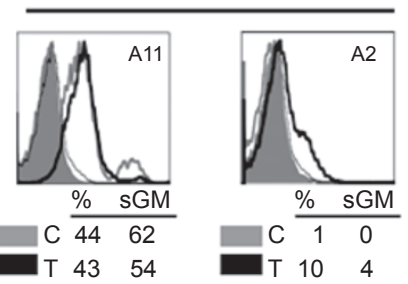

- $10 \quad 4$
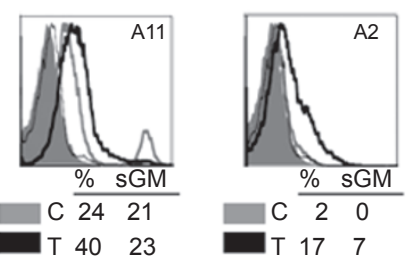

FL-2

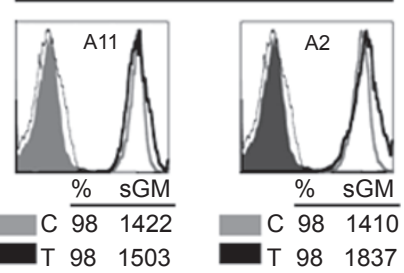

NSCM

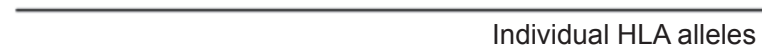

B

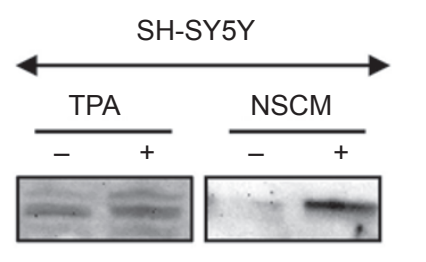

HLA-E
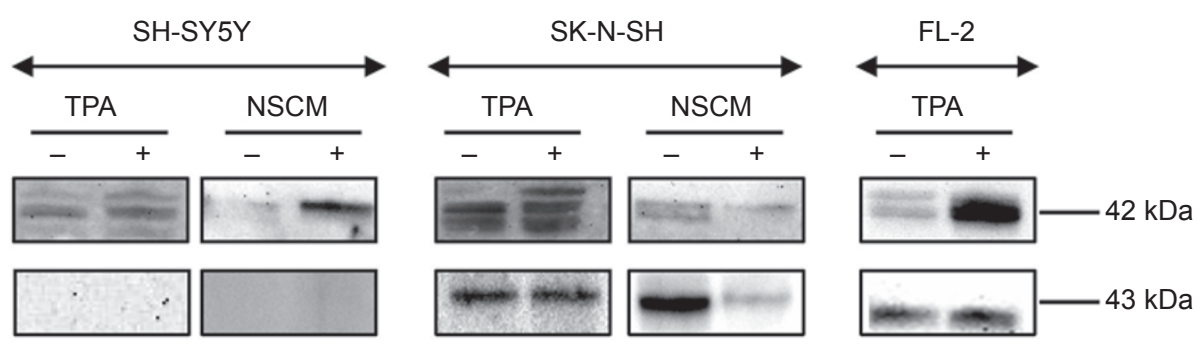

HLA-G
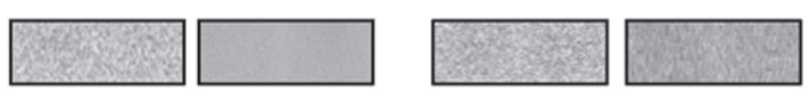

$\beta$-tubulin
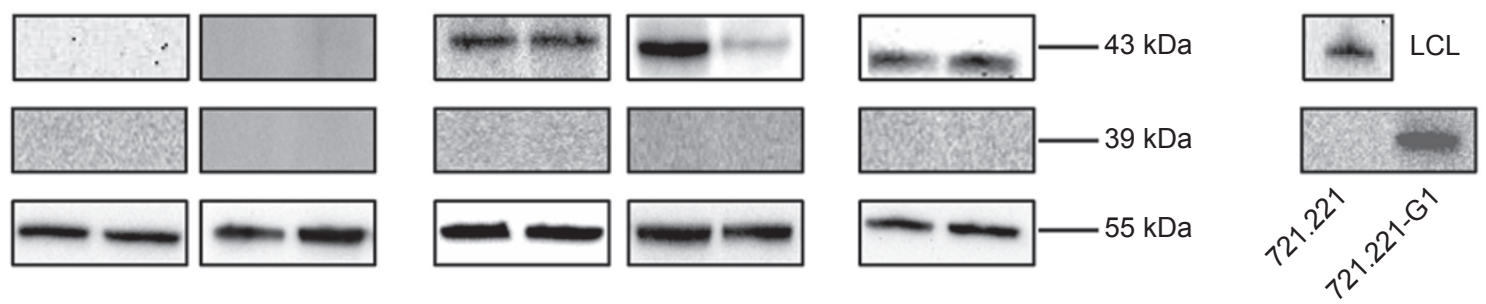

Figure 2 NB cell lines up-regulate MHC class I molecules in response to TPA and NSCM treatment. (A) Expression of the total pool of surface MHC class I complexes (HLA-ABC) or individual alleles (HLA-A2 and HLA-A11) was assessed in untreated (thick gray line) or DA-treated (thick black line) NB cell lines. Antibodies of relevant isotype were used as controls for untreated (filled histogram) or DA-treated (thin black line) cultures. The internal legends show the percent of positive cells (\%) and specific geomean (sGM) in control $(C)$ and treated $(T)$ cultures. (B) Expression of the total pool of MHC class I heavy chain, HLA-G or HLA-E in cell lysates of NB lines either treated with DA (+) or left untreated (-) was monitored by western blot. Total cell lysates of the BK-B5 LCL were used as a positive control for HLA-E expression. Specificity of the anti-HLA-G antibody was confirmed using total cell lysates of the HLA-G-transfected 721.221-G1 LCL and vector-transfected 721.221 LCL. Expression of $\beta$-tubulin was used as a control of sample loading. The corresponding molecular weights are indicated in kDa. 
A
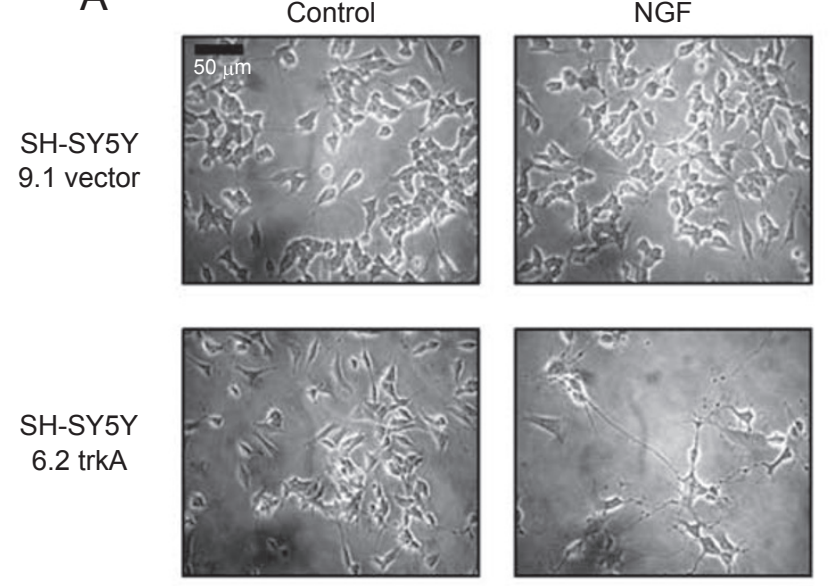

C

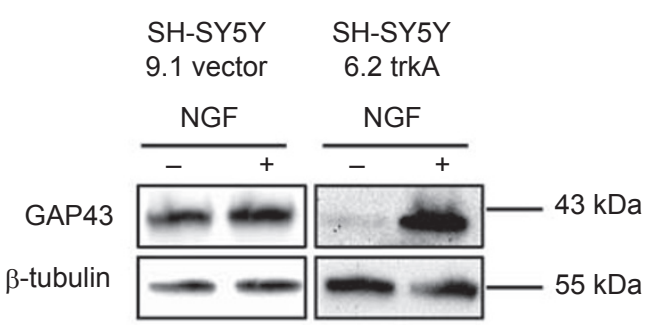

D
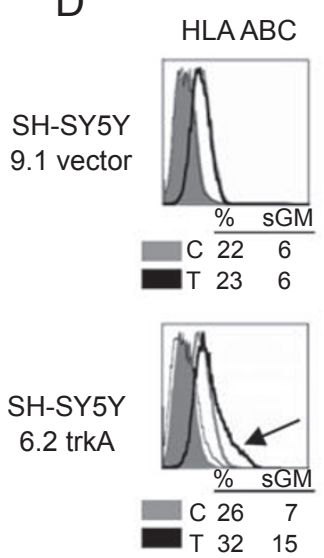

HLAA11
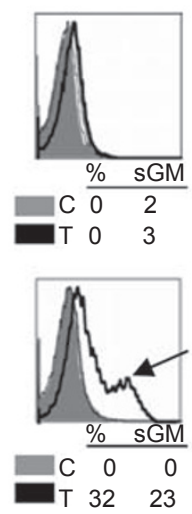

NGF

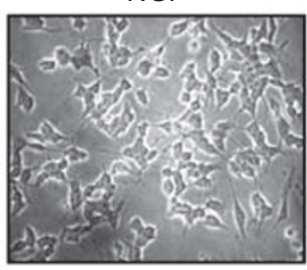

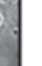

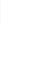

B

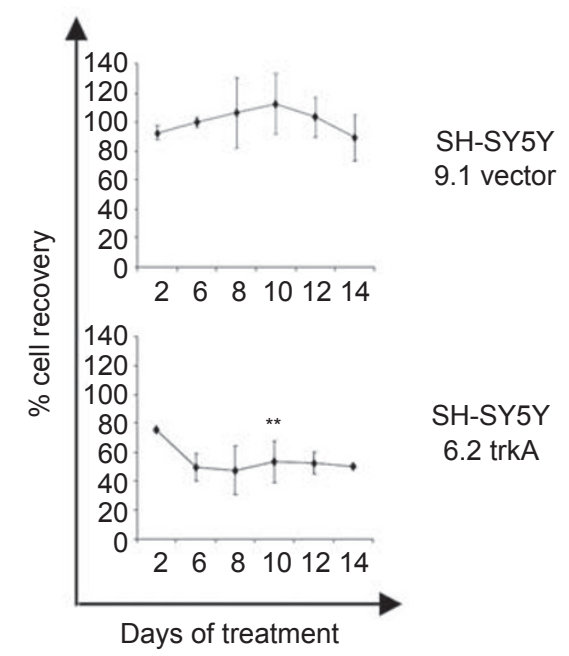

Figure 3 NGF treatment induces differentiation and MHC class I up-regulation in a trkA-positive, but not in a trkA-negative NB cell line. (A) Digital images of SH-SY5Y transfected with either trkA (clone 6.2 trkA) or vector plasmid (9.1 vector) were acquired after 6 days of treatment with NGF by a charge-coupled device attached to a phase-contrast microscope. Bar $=50$ $\mu \mathrm{m}$. (B) Numbers of trypan-blue negative NB cells in cultures either treated with NGF or left untreated were calculated at the indicated days of treatment. Cell recovery in NGF exposed cultures was calculated relative to that observed in control cultures and expressed in percent. Data from three or more experiments are presented for each time point as mean \pm SD. The value $^{* *}(P<0.01)$ demonstrates significant difference obtained on day 10. (C) Expression of GAP43 in total cell lysates of 9.1 vector and 6.2 trkA cells either treated with NGF (+) or left untreated (-) was monitored by western blot on day 8 of treatment. Membranes were subsequently stripped and re-probed for the expression of $\beta$-tubulin (control of sample loading). The corresponding molecular weights are indicated in $\mathrm{kDa}$. (D) Expression of the total pool of surface MHC class I complexes (HLA-ABC) and the individual allele HLA-A11 was assessed in untreated (thick grey line) and NGF-treated (thick black line and indicated by the arrow) NB cell lines at day 10 of treatment. Relevant isotype antibodies were used as controls for untreated (filled histogram) or NGF-treated (thin black line) cultures. The internal legends show the percent of positive cells (\%) and specific geomean (sGM) in control (C) and treated (T) cultures. Expression of the total pool of MHC class I heavy chain in cell lysates of cells either treated with NGF (+) or left untreated (-) was monitored by western blot at day 8 of treatment. Expression of HLA-G or HLA-E in total cell lysates was detected at day 8 using specific antibodies. The corresponding molecular weights are indicated in $\mathrm{kDa}$. The specificity of HLA-E- and HLA-G-specific antibodies was confirmed as described in the legend to Figure 2. 
Tumor cell differentiation promotes formation of conjugates between NB cells and effector lymphocytes

The formation of immune conjugates is a crucial event in target cell recognition by cytotoxic lymphocytes [15]. Expression of the intercellular adhesion molecule ICAM-1, which is known to play a critical role in formation of conjugates between killer lymphocytes and their targets, was examined prior to and after induction of NB differentiation. The steady-state levels of surface ICAM-1 were low (on SH-SY5Y and SK-N-SH) or moderate (FL-2, data not shown), but readily induced in response to TPA or NSCM in the course of differentiation (Figure 4A). In agreement with this, trkA-transfected, but not vector-transfected SH-SY5Y cells increased ICAM-1 levels by almost 4-fold in response to NGF (Figure 4B). Up-regulation of ICAM-1 on differentiated NBs was paralleled by enhanced rate of immune-conjugate formation, which was observed in different lines and under different conditions of differentiation (Figure 4C and Supplementary information, Figure S1).

\section{Differentiated NB cell lines are more efficiently killed by CTLs and NK cells}

Elimination of tumors by effector lymphocytes can be executed via two distinct routes: death-receptor-mediated pathway and granule-mediated exocytosis. The latter depends on the quality and quantity of the MHC class I complexes at the cell surface of tumor cells, when CTLs act as effectors [11]. We examined the susceptibility of NB cell lines to lysis by MHC class I-restricted HLAA2-specific allogeneic CTLs, and found that HLA-A2positive NB lines differentiated by TPA and NSCM were more efficiently killed by these effectors. The difference in CTL-mediated lysis (Figure 5A) was detected in spite of only marginal up-regulation of surface HLA-A2 (Figure 2A) in response to these differentiating stimuli. Although up-regulation of MHC class I expression may be inhibitory for NK cell activation, differentiated NBs were also more efficiently recognized by at least two of three tested NK lymphoma cell lines in $4 \mathrm{~h}{ }^{51} \mathrm{Cr}$-release assays (Figure 5B), which usually reflect the outcome of granule-mediated exocytosis. This finding prompted us to search for molecular mechanisms causing enhanced lysis of differentiated NB lines via granule-mediated exocytosis, and we found that human purified granzyme $\mathrm{B}$, the main apoptosis-inducing component of the cytolytic granules, binds more readily to the surface of differentiated NB cells as compared to their non-differentiated counterparts (Figure 5C).

Differentiation of ex vivo isolated NB tumors increases their immunogenicity
Next, we analyzed ex vivo isolated NB tumor cells designated by us as NB-KI-4 and NB-KI-6 for their ability to differentiate in response to TPA, NSCM or NGF (Figure 6), since the NB-KI-6 tumor appeared to be trkA-positive as revealed by immunostaining and subsequent flow cytometry (data not shown). Freshly isolated NB cells responded to differentiation stimuli by changing their cell morphology (Figure 6A) and up-regulating GAP43 (Figure 6C). In contrast to the cell lines, primary NB cells persisted as a suspension of spheroids; however, both TPA and NSCM promoted their adherence to the plastic surfaces. Clear extensive neurite outgrowth could be seen at the periphery of the spheroids. Morphological changes in response to differentiating signals are exemplified by NB-KI-4 tumor cells (Figure 6A). Similar to NB cell lines, surface MHC class I (Figure $6 \mathrm{~B}$ ), the total pool of class I heavy chains (Figure 6C) and ICAM-1 (Figure 6D) were induced in the course of differentiation in freshly isolated NB cells. Similar to NB cell lines, no detectable HLA-G expression was observed by western blot in ex vivo analyzed NB cells either prior to or after differentiation (Figure 6C). HLA-E was expressed by both tumors and its expression remained largely unchanged in the course of differentiation of NBKI-6, whereas NB-KI-4 strongly down-regulated HLA-E expression when differentiated with NSCM (Figure 6C). These changes were accompanied by the increased ability to assemble immune conjugates as compared to nondifferentiated tumor cells (Figure 6D).

\section{Discussion}

Due to its severe side effects such as general toxicity and immunosuppression, as well as the development of multi-drug resistance, classical chemotherapy based on destruction of tumor cells requires complementary approaches which do not entail cell death (reviewed in [1]). Differentiation therapy fulfills this requirement and various DAs demonstrated efficacy in clinical tests, e.g. TPA in the treatment of hematologic malignancies $[16,17]$ and retinoids as a component of minimal residual disease treatment in NB patients [4].

In the case of late-stage NB, even aggressive chemotherapy with autologous hematopoietic stem cell rescue does not preclude poor prognosis [18]. A number of other complementary therapeutic approaches, such as antiangiogenic and apoptosis-inducing agents, gene therapy and immunotherapy (reviewed in [19]), have shown some success, but only in pre-clinical models of NB, whereas retinoid-based differentiation therapy already demonstrated effectiveness in clinical settings (reviewed in [4]). 
A

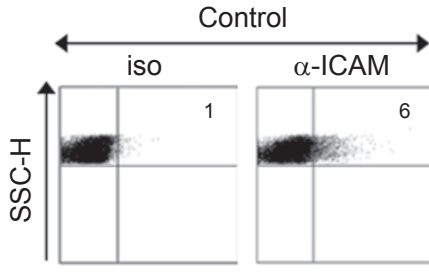

TPA

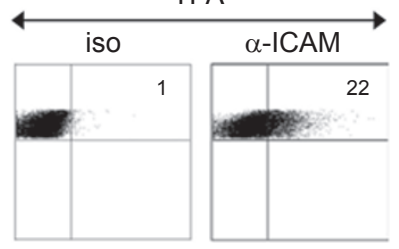

NSCM

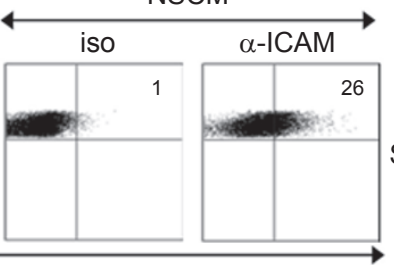

SH-SY5Y
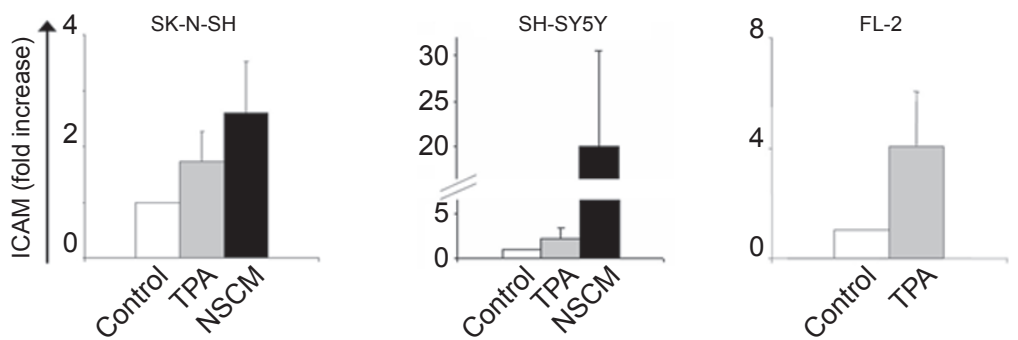

B
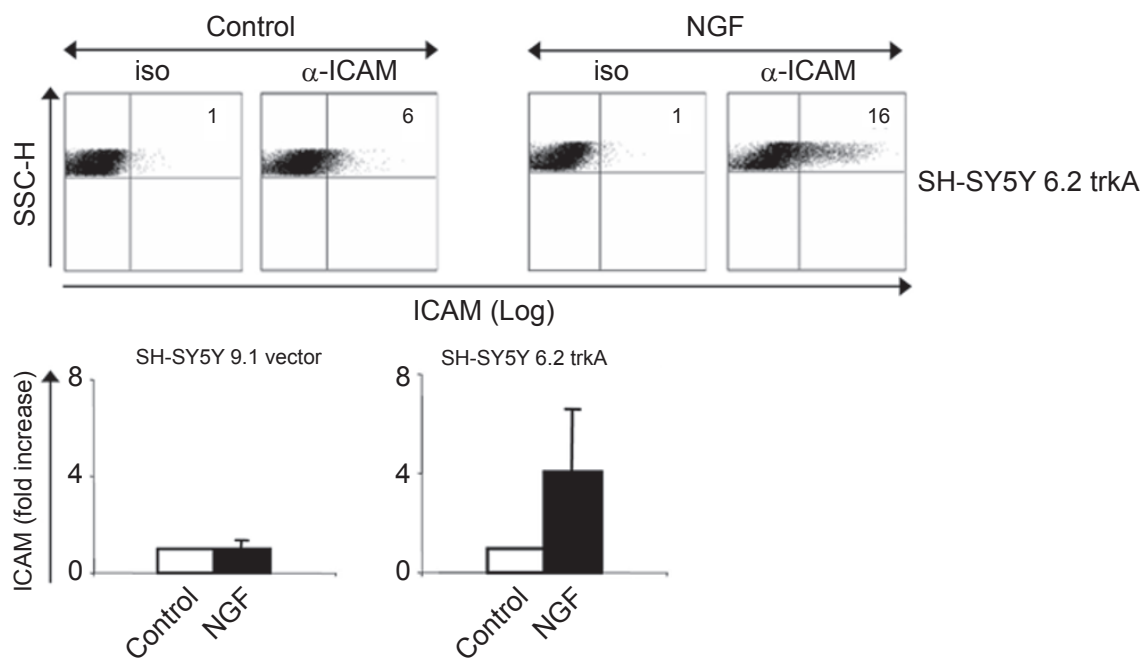

C
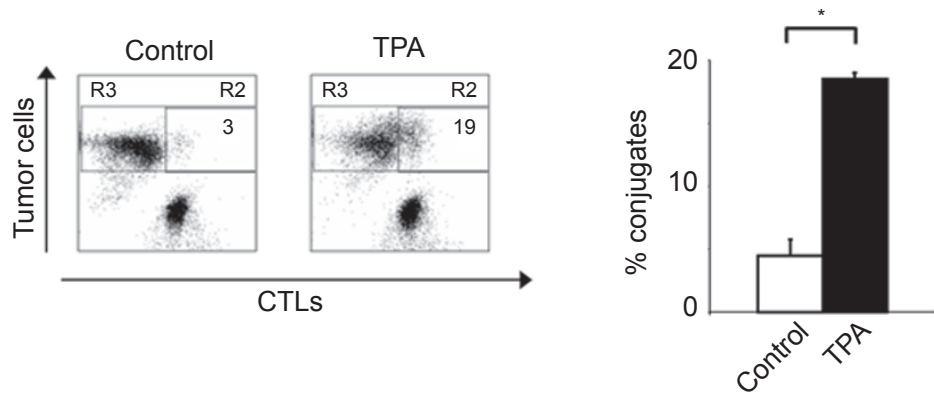

Figure 4 Differentiated NB cells up-regulate ICAM-1 at the cell surface and more readily form conjugates with effectors. (A) FACS analysis of surface ICAM-1 expression in untreated (control), TPA- or NSCM-treated SH-SY5Y NB cell line was performed on day 3 and 12 of treatment, respectively. Numbers indicate percentages of ICAM-1-positive cells. Bars show increase in ICAM-1 expression upon TPA or NSCM treatment of indicated NB cell lines calculated as fold increase compared to levels observed in untreated cells. (B) FACS analysis of surface ICAM-1 expression in untreated (control) or NGF-treated SHSY5Y transfected with trkA (clone 6.2 trkA) was performed on day 10 of treatment. Numbers indicate percentages of ICAM1-positive cells. Bars show increase in ICAM-1 expression upon NGF treatment of trkA- and vector-transfected NB cells calculated as fold increase compared to levels observed in untreated cells. (C) FACS analysis of conjugate formation between the FL-2 cell line, either untreated (control) or TPA-treated, and GLC CTLs (all labeled with fluorescent dyes as described in Materials and Methods) after $30 \mathrm{~min}$ of incubation at $+37^{\circ} \mathrm{C}$. Numbers indicate percentage of conjugates, defined as doubledye-positive cells (R2 region) of the total number of tumor cells (R3 region). Percentage of FL-2 cells forming conjugates with effectors prior to and after treatment with TPA is summarized in the graph (right panel). Mean \pm SD of three experiments is shown. The value * $(P<0.05)$ demonstrates significant difference. 
A
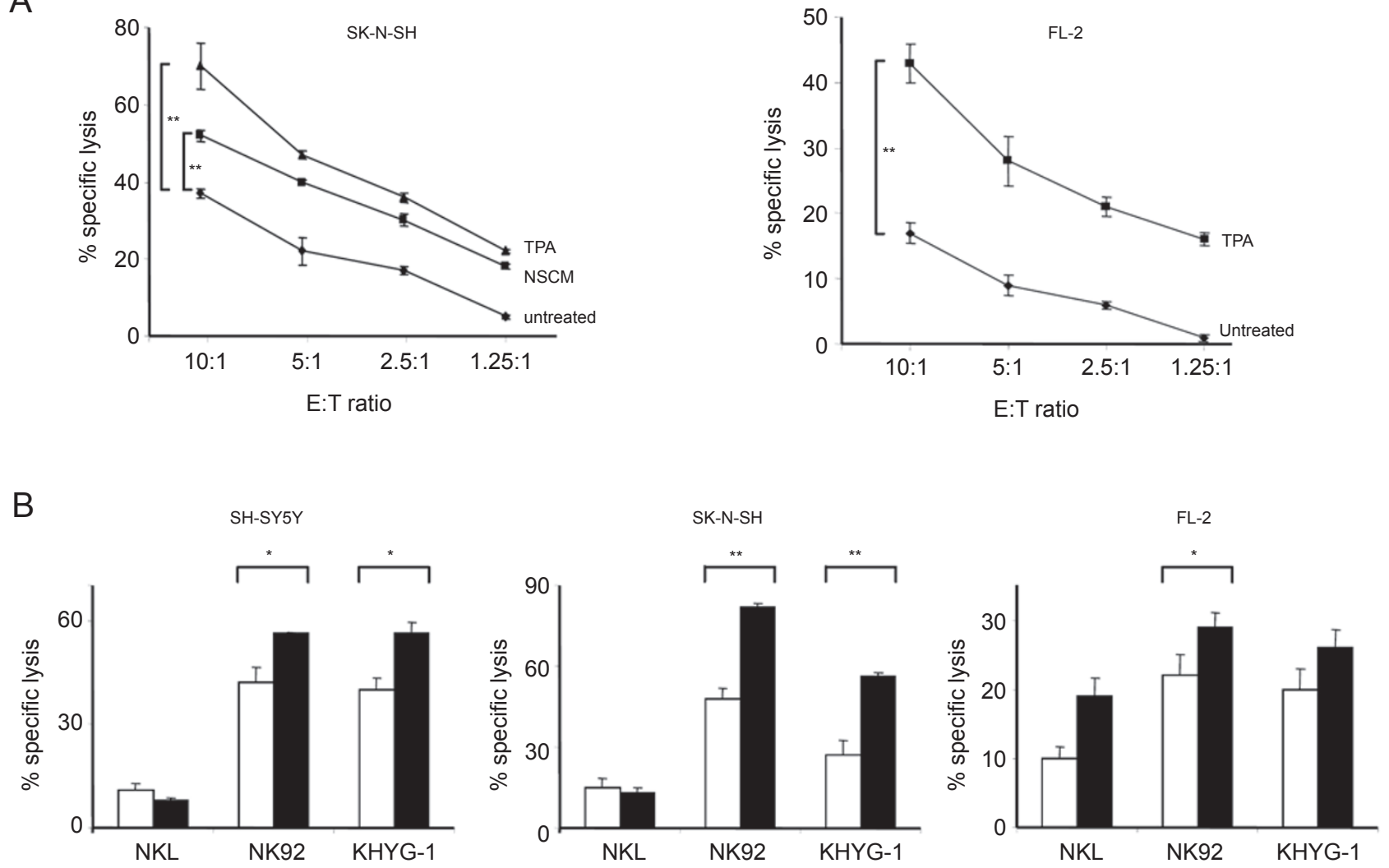

C

SH-SY5Y

Control

TPA
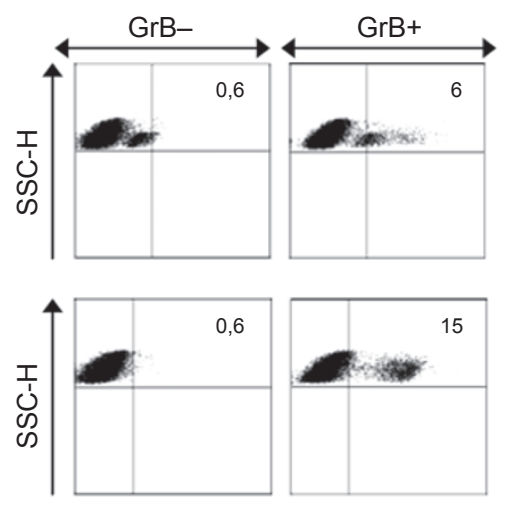

SK-N-SH

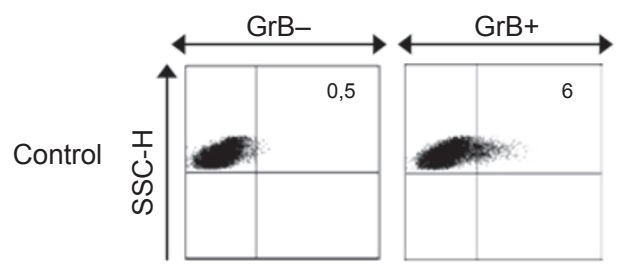

NSCM
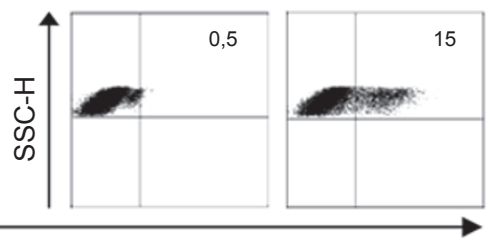

Granzyme B (Log)

Figure 5 Differentiating NB lines are more efficiently lysed by cytotoxic lymphocytes and bind more granzyme $B$ at the cell surface. (A) HLA-A2-positive NB lines SK-N-SH and FL-2 either untreated or treated with TPA or NSCM for 6 days were used as targets for allogeneic HLA-A2-specific CTLs in a $4 \mathrm{~h} \mathrm{Cr}$-release assay at the effector-to-target ratios indicated in the figure. (B) SH-SY5Y, SK-N-SH and FL-2 cell lines either untreated (white bars) or treated with TPA for 6 days (black bars) were used as targets for NK lines NKL, NK92 and KHYG-1 in a $4 \mathrm{~h}$ Cr-release assay at 1:5 effector to target ratio. Mean \pm SD of three experiments is shown. Values * $(P<0.05)$ and ${ }^{* *}(P<0.01)$ demonstrate significant differences. (C) Binding of human purified granzyme B (see Materials and Methods) to the surface of NB cell lines either untreated (control, upper panels) or treated with TPA or NSCM (lower panels) for 3 or 11 days, respectively, was monitored by flow cytometry after staining with granzyme B-specific antibody. Samples kept under identical conditions, but not exposed to granzyme B, were used as controls (GrB-). One representative of three performed experiments is shown in the figure. 
A

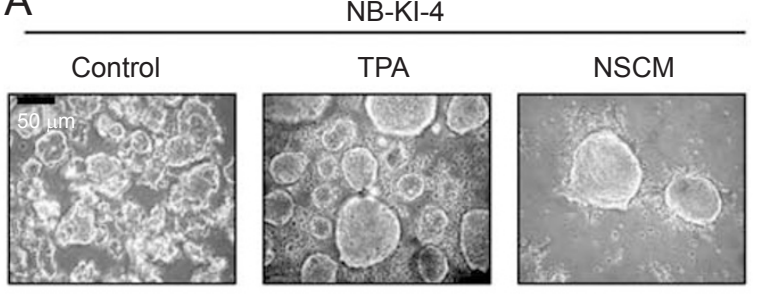

C

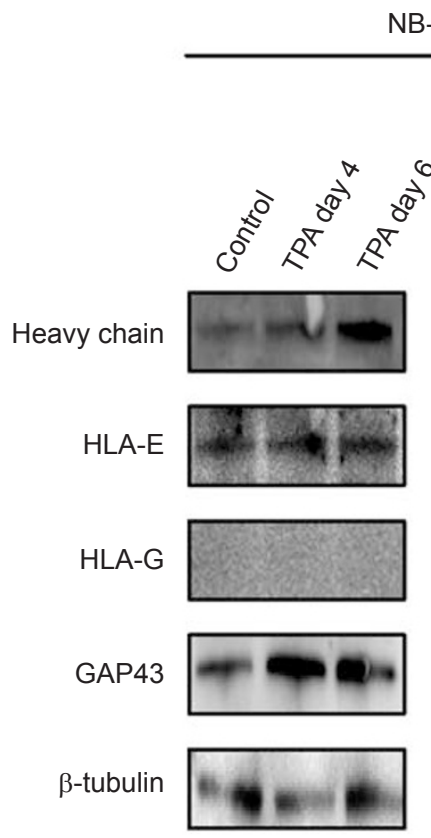

NB-KI-4
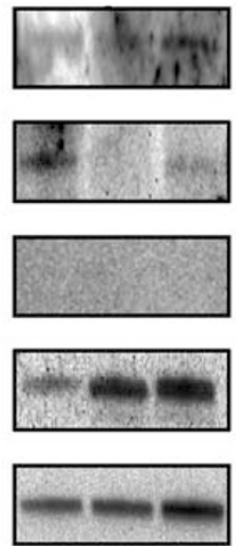

B

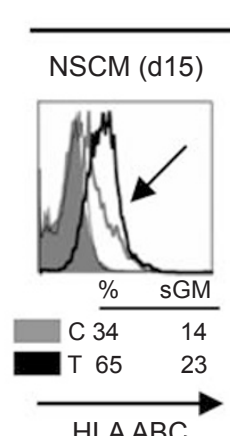

NB-KI-4

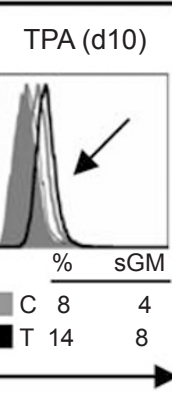

HLA ABC
NB-KI-6 TPA (d6)

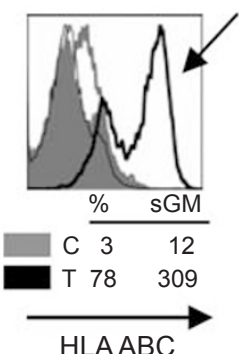

NB-KI-6

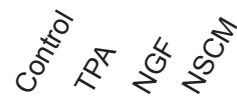
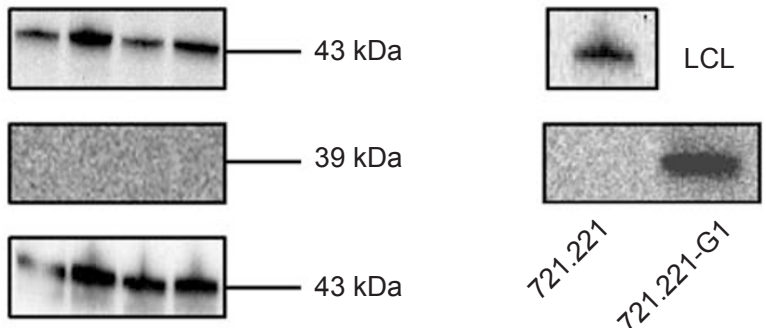

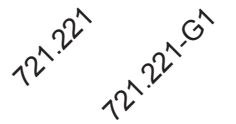

$-55 \mathrm{kDa}$

NB-KI-4
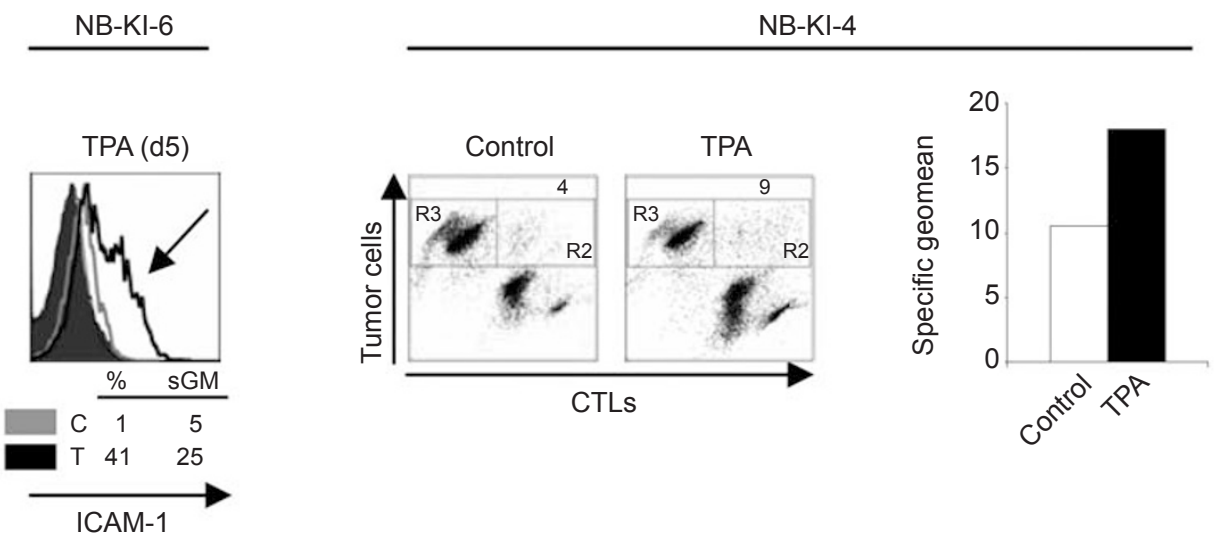

Figure 6 Freshly isolated neuroblastoma cells are susceptible to differentiation-inducing agents and change their immunogenicity. Different DAs were used for different tumor samples, as indicated in the figure. (A) Morphological changes in the tumor NB-KI-4 induced by TPA (day 4 of treatment) and NSCM (24 h of treatment) were monitored through a phase-contrast microscope and digital images were acquired by a charge-coupled device. Bar $=50 \mu \mathrm{m}$. (B) Expression of the total pool of surface MHC class I complexes (HLA-ABC) was assessed in untreated (thick gray line) and DA-treated (thick black line and indicated by the arrow) freshly isolated NB-KI-4 and NB-KI-6 tumor cells. Relevant isotype antibodies were used as controls for untreated (filled histogram) or DA-treated (thin black line) cells. The internal legends show the percent of positive cells (\%) and 
We have previously demonstrated that retinoids induce expression of proteolytic and regulatory subunits of the immunoproteasome, increase the half-life of MHC class I complexes and enhance the sensitivity of NB cells to both MHC class I-restricted peptide-specific and HLA non-restricted lysis by CTLs [5]. Though we have demonstrated that the effects of retinoids on the MHC class I pathway are independent of IFN $\gamma$ and/or $\mathrm{TNF}_{\alpha}$ as intermediate messengers, it still remains unclear whether modulation of the immunogenicity of NB cells by retinoids is a reflection of a differentiated phenotype of the tumor or is a differentiation-independent effect of retinoids. To address this question, we have used (i) TPA, an agonist of the PKC signaling pathway, (ii) a combination of EGF, FGF and LIF $[20,21]$ and (iii) trkA stimulation by the high-affinity ligand NGF, all of which are known to induce neuronal differentiation in NB cells. All of these agents were able to trigger differentiation not only in NB cell lines but also in ex vivo analyzed NB tumor cells. The repertoire and magnitude of molecular changes observed in NB cells after treatment with DAs are summarized in Supplementary information, Table S1. In contrast to other NBs tested, the anti-proliferative effect exerted by NSCM on FL-2 cells was accompanied neither by an increase in GAP43 expression nor by the morphological changes in these cells, at least in the time-frame of our experimental procedures. Therefore, we cannot formally conclude that NSCM is capable of triggering differentiation in FL-2 cells. The molecular mechanisms for such an effect are outside the scope of the current study and need to be investigated further.

Whereas controversial data are available concerning the ability of the DAs used in our study to up-regulate MHC class I expression in cells of different origin [22-24], most reports indicate their capability to increase surface ICAM-1 levels [25-28]. However, none of the reports clearly linked these changes to a differentiated phenotype in NB cells. We found that changes in NB cell immunogenicity monitored in a kinetic time course of treatment with DAs never preceded, but usually always followed the differentiation-compatible features, such as growth arrest, altered cell morphology and up-regulation of GAP43. Interestingly, no significant increase in the expression of neuron-specific enolase (NSE) was observed in NB cells undergoing differentiation even at those time points of treatment when prominent morphological changes were detected (data not shown). This could be explained by the high steady-state levels of this protein as observed by western blot in the NB cells tested, which, probably, reflects the characteristic of the neuronal lineage.

Similar to retinoids, TPA, NSCM and NGF were capable of up-regulating the total pool of surface MHC class I complexes on NB cell lines (Figures 2A and 3D). However, in contrast to retinoids [5], just a slight, if any, up-regulation of HLA-A2 and -A11 was observed (Figure 2A). In the case of FL-2, treatment with TPA induced HLA-A2 more readily than HLA-A11, which might reflect the differences in generation of peptides capable of binding to and stabilizing selected individual HLA at the cell surface upon TPA treatment.

Nevertheless, more efficient CTL-mediated lysis was detected when NBs were differentiated with TPA and NSCM (Figure 5A). Antigen-independent adhesion mediated by molecular interactions such as LFA-1:ICAM-1 and CD2:LFA-3 is regarded as a critical step in the interaction between effectors and targets during the immune response [15]. The fact that ICAM-1 was up-regulated by NB cells in the course of differentiation (Figure 4A and $4 \mathrm{~B}$ ) can partially explain the outcome of the cytotoxicity. Indeed, we and others have previously observed that up-regulation of ICAM-1 expression facilitates more effective MHC-restricted and non-restricted cytotoxicity $[5,29]$. In agreement with these data, an increased ability of differentiated NB cells to form conjugates with the effector cells was also observed (Figure 4C). In addition, the up-regulation of surface ICAM-1 and enhanced conjugate formation by differentiated NBs may also explain

specific geomean (sGM) in control (C) and treated (T) cultures. (C) Expression of the total pool of MHC class I heavy chain, HLA-G or HLA-E in cell lysates either treated with DA (as indicated) or left untreated (control) was monitored by western blot. Expression of GAP43 was used as a marker for differentiation, and expression of b-tubulin was used as a control of sample loading. The corresponding molecular weights are indicated in kDa. The specificity of HLA-E- and HLA-G-specific antibodies was confirmed as described in the legend to Figure 2. (D) Expression of surface ICAM-1 in untreated (thick gray line) and TPA-treated (thick black line indicated by the arrow) NB-KI-6 tumor. Relevant isotype antibodies were used as controls for untreated (filled histogram) or TPA-treated (thin black line) cultures. The internal legend shows the percent of positive cells (\%) and specific geomean (sGM) in control $(C)$ and treated (T) cultures. Conjugate formation between NB-KI-4, either untreated (control) or TPA-treated, and GLC CTLs (all labeled with fluorescent dyes as described in Materials and Methods) after 30 min of incubation at $+37{ }^{\circ} \mathrm{C}$. Numbers indicate percentage of conjugates defined as double-dye-positive cells (R2 region) of the total number of tumor cells (R3 region). The graph shows data obtained from staining with ICAM-1-specific antibody on the same cultures. Specific geomean is shown for ICAM-1-positive cells. 
the enhanced susceptibility of these cells to NK-mediated lysis (Figure 5B), as previously observed in other models [30]. Finally, the enhanced binding of a main apoptosisinducing component of the cytolytic granules, the granzyme B molecule, to the surface of differentiated as compared to undifferentiated NB cells (Figure 5C), may also contribute to the increased CTL- and NK-mediated cytotoxicity. The molecular mechanism responsible for the increased granzyme B binding remains to be uncovered.

Importantly, the DAs used in our study triggered differentiation-associated changes, such as morphological alterations and GAP43 up-regulation, not only in established NB cell lines but also in ex vivo isolated tumor cells (Figure 6A and 6C and data not shown), which were subjected to the treatment within 4-24 h post surgical intervention. In agreement with the data obtained with NB lines, ex vivo isolated differentiated NB cells accumulated MHC class I complexes and ICAM-1 at the surface (Figure 6B and 6D). Due to the limited amount of available patient material and HLA mismatch between the tumors and effectors, we were not able to perform direct cytotoxicity assays. However, the increased ability of TPA-differentiated NB-KI-4 tumor to form immune conjugates with GLC CTLs paralleled by an increase in surface density of ICAM-1 in cells expressing this molecule (Figure 6D) prompts us to speculate that differentiation may increase the susceptibility of NBs to lysis by CTLs.

Non-classical HLAs, such as HLA-E and -G, at the surface of tumor cells may trigger inhibitory signals in both NK cells and activated CTLs [31, 32]. In contrast to previously published data on the ability of LIF to stimulate the HLA-G promoter and up-regulate HLA-G expression $[33,34]$, neither NB cell lines nor tumor cells freshly isolated from NB patients up-regulated HLA-G expression when differentiated with LIF-containing NSCM (Figures $2 \mathrm{~B}$ and $6 \mathrm{C}$, respectively). It is well established that the signal sequence peptide from HLA-G is one of the main epitopes stabilizing HLA-E at the surface [35]. At least two of the NB lines analyzed in our study, SK-N-SH and FL-2, as well as both freshly isolated tumor cell specimens expressed HLA-E at the protein level in total cell lysates (Figures $2 \mathrm{~B}$ and $6 \mathrm{C}$, respectively). Whereas the expression of HLA-E was not affected by TPA in SK-NSH, FL-2, NB-KI-4 or NB-KI-6 cells, the NSCM-driven differentiation led to a decrease of the HLA-E levels in both SK-N-SH and NB-KI-4 cells (Figures 2B and 6C, respectively). This was paralleled by a decrease of the total pool of MHC class I heavy chain in the cell lysates of NSCM-treated SK-N-SH cells (Figure 2B), suggesting that HLA-E molecules constituted a large proportion of the total pool of heavy chain in these cells. Interestingly, neither of the treatments modulated HLA-E expression in lysates from NB-KI-6 cells (Figure 6C). The functional significance of HLA-E modulation in the course of NB differentiation deserves special attention. It is still unclear whether HLA-E is expressed at the surface of NB cells, because at present there is no commercially available HLA-E-specific antibody suitable for flow cytometry. Our data also prompt us to speculate that, in the absence of HLA-G, HLA-E can be stabilized by peptides derived from other molecules, the expression of which undergoes changes in the course of tumor differentiation.

In our ${ }^{51} \mathrm{Cr}$-release assays we used allogeneic HLAA2-specific CD8+ CTLs. Since allo-specific CTLs recognize MHC-peptide complexes both in a peptide-dependent and -independent manner [36], the increased recognition by A2-specific CTLs may reflect an increase of HLA-A2 expression at the cell surface and/or improved production and presentation of certain HLA-A2 restricted peptide epitopes. It is still to be established whether or not the repertoire of peptide epitopes presented by the MHC class I complexes on differentiated NBs differs from that on untreated cells. This issue is of special interest, because it may directly influence the choice of the T-cell receptors selected for adoptive CTL transfer in NB patients. There is already an indication that the NGF-induced neurite outgrowth in differentiating cells is accompanied by a coordinated up-regulation of Ub-conjugating activity and elevated levels of ubiquitylated proteins [37], thus suggesting alterations in the proteolysis and generation of peptide epitopes. Therefore, identification of the tumor-specific MHC class I-restricted peptides presented on the surface of differentiated NBs represents an important and challenging task.

Differentiation therapy of tumors is believed to inhibit tumor cell proliferation and eventually lead to physiological elimination of differentiated "ex-malignant" cells. Nonetheless, it is still unclear to what extent this scenario is applicable to NB patients. Additional targeting of differentiated NB cells is desirable and may be executed by cytotoxic lymphocytes. Though we were not able to include high-risk NB patients in this study, and supplementary analysis on the effect of DAs on tumor cells derived from these patients is still warranted, our data point towards the possibility to successfully combine immunotherapy and differentiation therapy in NB patients, encouraging additional studies on the effect of DAs on CTL- and NK-mediated recognition of tumor cells of different origins.

\section{Materials and Methods}

\section{Cell culture media and supplements}

IMDM, AIM-V, DMEM:F12, B-27 Supplement and FCS were 
from GibcoBRL, Life Technologies (Grand Island, NY, USA).

\section{Tumor cell lines}

The NB cell lines SH-SY5Y and SK-N-SH were purchased from the American Type Culture Collection (ATCC, Manassas, VA, USA). The FL-2 cell line, a subclone of SH-SY5Y, was provided by Dr M Ifversen (Rigshospitalet, Copenhagen, Denmark). The NK cell line NK-92 was obtained from ATCC, and KHYG-1 was obtained from The Human Science Research Resources Bank (JCRB0156; Tokyo, Japan), respectively; NKL was kindly provided by Dr Jonas Sundback (Microbiology and Tumor and Cell Biology Department, Karolinska Institutet, Sweden). The cell lines were maintained in IMDM supplemented with $10 \%$ heat-inactivated FCS, $100 \mathrm{IU} / \mathrm{mL}$ penicillin and $100 \mathrm{mg} / \mathrm{mL}$ streptomycin (complete medium). The generation and propagation of SH-SY5Y 6.2 (trkA transfectant) and SH-SY5Y 9.1 (vector transfectant) were described previously [12].

\section{Patient material}

Tumor cells NB-KI-4 and NB-KI-6 were isolated from NB patients undergoing surgery at Astrid Lindgren's Children Hospital, Stockholm, Sweden. Tumor material was obtained and examined after informed consent was received from each subject's guardian. NB-KI-4 tumor sample 1 was obtained from the kidney, whereas samples 2 and 3 of the same tumor were obtained from tumor mass spread to the diaphragm region of a 14-months-old male patient with non-differentiated stage II NB. NB-KI-6 tumor sample was obtained from the kidney region of a 5-months-old female patient and contained both differentiated ganglionic and non-differentiated stage I NB cells. Tumor cells were propagated in AIM-V containing $10 \%$ heat-inactivated FCS, $100 \mathrm{IU} / \mathrm{mL}$ penicillin, $100 \mu \mathrm{g} / \mathrm{mL}$ streptomycin and $20 \mu \mathrm{g} / \mathrm{mL}$ of cyproxin (complete AIM-V).

\section{Induction of differentiation}

The following substances were used as DAs: phorbol ester, tetradecanoylphorbol acetate (TPA) purchased from Sigma (St Louis, MO, USA); recombinant human $\beta$-nerve growth factor ( $\beta$-NGF) purchased from R\&D systems (Abingdon, UK); recombinant human EGF and recombinant human basic FGF from Peprotech (London, UK); recombinant leukemia inhibitory factor (LIF) from Chemicon (Hampshire, UK). Treatment with the TPA was performed at a final concentration of $16 \mathrm{nM}$ in complete medium for the indicated periods of time, usually 3 or 6 days. Cells kept in complete medium containing the corresponding amount of $95 \%$ ethanol are thereafter referred to as "control". Treatment further referred to as NSCM [9] was performed in DMEM:F12 containing B-27 (1:50), $20 \mathrm{ng} / \mathrm{mL}$ of LIF, $20 \mathrm{ng} / \mathrm{mL}$ of EGF and $20 \mathrm{ng} / \mathrm{mL}$ of FGF for 8-12 days. Replenishment of EGF and FGF was performed at day 4 and 8 . Treatment with NGF at a final concentration of $100 \mathrm{ng} / \mathrm{mL}$ was done in complete medium for 8-10 days. Replenishment of NGF was performed every 2-4 days. For induction of differentiation in freshly isolated NBs, tumor cells were treated with either $16 \mathrm{nM}$ TPA for 5-10 days or NSCM for 8-12 days. TrkA-expressing NB-KI-6 cells were treated with $200 \mathrm{ng} / \mathrm{mL}$ of NGF in complete AIM-V for 6 days.

\section{Antibodies and reagents}

Mouse monoclonal antibody specific for $\beta$-tubulin was purchased from Sigma (St Louis, MO, USA); mouse monoclonal an- tibody specific for human trkA was purchased from R\&D systems (Abingdon, UK). The R-phycoerythrin (RPE)-conjugated HLAABC-specific antibody (clone W6/32), RPE-conjugated mouse IgG2a isotype antibody, mouse IgG1 isotype antibody, RPE-conjugated rabbit anti-mouse $\mathrm{F}(\mathrm{ab})_{2}$ fragments, horseradish peroxidase (HRP)-conjugated rabbit anti-mouse IgG and goat anti-rabbit IgG were from DakoCytomation (Solna, Sweden). The allophycocyanin (APC)-conjugated ICAM-1-specific antibody, APC-conjugated mouse IgG1 isotype control, APC-conjugated goat anti-mouse IgGs, FITC-conjugated human CD56-specific antibody and FITCconjugated mouse IgG2b isotype control were purchased from BD Pharmingen (San Diego, CA, USA). Hybridomas producing the HLA-A11-specific antibody (clone HB-164) and the HLAA2-specific antibody (clone HB-54) were purchased from the ATCC. Total mouse serum was prepared by the animal facility at the Microbiology and Tumorbiology Center, Karolinska Institutet. Rabbit polyclonal serum specific for human class I heavy chain was a kind gift from Dr Hidde Ploegh (Department of Pathology, Harvard Medical School, Boston, MA, USA). The anti-GAP43 antibody was from Abcam (Cambridge, UK). The HLA-E-specific antibody (clone MEM-E/02), the HLA-G-specific antibody (clone MEM-G/01) and human purified granzyme B were from ALEXIS Corporation (Lausen, Switzerland). The RPE-conjugated mouse monoclonal antibody specific to human granzyme B was purchased from Serotec (Hamar, Norway). TFL2 and TFL4 dyes were obtained from Grantoxilux ${ }^{\circledR}$ kit (Oncoimmunin Inc., Gaithersburg, MD, USA).

\section{Assessment of differentiation}

Numbers of trypan blue-negative NB cells were calculated in control and DA-treated cell cultures at indicated time points of treatment. Expression of the neuronal marker gene GAP43 was monitored by western blot (see below). Changes in cell morphology were monitored using a phase-contrast microscope and digital images were acquired by a charge-coupled device at the indicated days of treatment with DA.

\section{Western blot analysis}

Western blot analysis was performed using a Multiphor II Electrophoresis System and ExelGel SDS homogenous precast gels (Amersham Pharmacia Biotech AB, Uppsala, Sweden). Total cell lysates obtained from $10^{5} \mathrm{NB}$ cells cultured in complete medium or medium containing DA under the conditions described above were resolved by SDS-PAGE followed by transfer onto a polyvinylidene difluoride membrane (Millipore AB, Sundbyberg, Sweden). Membranes were blocked in PBS containing 5\% milk and $0.1 \%$ Tween-20 and probed with the relevant specific antibody diluted as indicated by the manufacturer's protocol. Rabbit antimouse IgG or goat anti-rabbit IgG conjugated to HRP were used as secondary antibodies at a 1:5000 dilution. Enhanced chemiluminescence was used to visualize the reaction (Amersham Pharmacia Biotech AB).

\section{FACS analysis}

For assessment of the total surface pool of MHC class I complexes, NB cells cultured in either complete or DA-containing medium (as stated above) were incubated with the RPE-labeled HLA-ABC-specific antibody W6/32 or RPE-labeled IgG2a isotype control. The level of surface ICAM-1 was evaluated after incuba- 
tion with APC-labeled anti-ICAM-1 antibody or APC-labeled mouse IgG1 isotype control. Surface expression of HLA-A2 and HLA-A11 was monitored after incubation with either the relevant specific antibody or total mouse serum as a negative control followed by incubation with APC-conjugated anti-mouse IgGs as secondary antibodies. Tumor cells in freshly isolated NB samples were defined as "CD56-bright" as described previously [38] and usually constituted the vast majority of the tumor sample preparations.

\section{Conjugation assay}

Conjugation assays were performed as described previously [29]. Briefly, both NB cells, either untreated or treated with DA, and effectors (GLC CTLs) were fluorescently labeled with either TFL2 or TFL4 dyes, incubated at $1: 1$ ratio either at $+37{ }^{\circ} \mathrm{C}$ for $30 \mathrm{~min}$ or on ice (control for specificity of conjugate formation), and subsequently analyzed by FACS. Conjugates were defined as double-dye-positive cells.

\section{Granzyme B binding assay}

NB cell lines, either untreated or treated with DA under the conditions described above, were washed twice in ice-cold PBS $/ 0.1 \%$ BSA prior to incubation with $6 \mathrm{ng}$ of human purified granzyme B in a volume of $20 \mu 1$. Equal volumes of PBS $/ 0.1 \%$ BSA were added to parallel samples (designated as control). Following incubation in a v-bottom polypropylene 96-well plate for $45 \mathrm{~min}$ on ice, $2 \times 10^{5}$ tumor cells were extensively washed in icecold PBS $/ 0.1 \%$ BSA, and exposed to RPE-labelled anti-human granzyme B antibody $(10 \mu \mathrm{g} / \mathrm{mL})$ for $45 \mathrm{~min}$ on ice. Cells were extensively washed prior to FACS analysis.

\section{Cytotoxicity assays}

Standard $4 \mathrm{~h}{ }^{51} \mathrm{Cr}$-release assays were performed as described previously [39]. Briefly, NB cell lines were treated with DA (16 nM of TPA or NSCM for 6 days) or left untreated, labeled with $\mathrm{Na}^{51} \mathrm{CrO}_{4}$ $\left(0.1 \mu \mathrm{Ci} / 10^{6}\right.$ cells at $37^{\circ} \mathrm{C}$ for $\left.1 \mathrm{~h}\right)$ and incubated with either HLA-A2 restricted allogeneic CTLs or NK cell lines NKL, NK-92 or KHYG-1 in triplicates for $4 \mathrm{~h}$ at $37{ }^{\circ} \mathrm{C}$ at indicated effector-totarget ratios. ${ }^{51} \mathrm{Cr}$-release in the supernatants was measured by a $\gamma$-counter (Wallac Sverige AB, Stockholm, Sweden).

\section{Statistical analysis}

Mean values of at least three experiments were used to calculate standard deviations. Statistical significance was analyzed using bifactorial variance analysis for comparison of differentiated versus non-differentiated cells.

\section{Acknowledgments}

We would like to thank Dr V Levitsky for providing CTL clones. This work was supported by the Swedish Children's Cancer Foundation, the Swedish Cancer Society, the Cancer Society of Stockholm, the King Gustav $\mathrm{V}^{\text {th }}$ Jubilee Fund and the Swedish Research Council.

\section{References}

1 Marchal JA, Rodriguez-Serrano F, Campos J, et al. Differen- tiation: an encouraging approach to anticancer therapy. Ital $J$ Anat Embryol 2006; 111:45-64.

2 Abelev GI, Lazarevich NL. Control of differentiation in progression of epithelial tumors. Adv Cancer Res 2006; 95:61-113.

3 Edsjo A, Holmquist L, Pahlman S. Neuroblastoma as an experimental model for neuronal differentiation and hypoxiainduced tumor cell dedifferentiation. Semin Cancer Biol 2006.

4 Altucci L, Gronemeyer H. The promise of retinoids to fight against cancer. Nat Rev Cancer 2001; 1:181-193.

5 Vertuani S, De Geer A, Levitsky V, et al. Retinoids act as multistep modulators of the major histocompatibility class I presentation pathway and sensitize neuroblastomas to cytotoxic lymphocytes. Cancer Res 2003; 63:8006-8013.

6 Martin RF, Beckwith JB. Lymphoid infiltrates in neuroblastomas: their occurrence and prognostic significance. J Pediatr Surg 1968; 3:161-164.

7 Hedborg F, Bjelfman C, Sparen P, Sandstedt B, Pahlman S. Biochemical evidence for a mature phenotype in morphologically poorly differentiated neuroblastomas with a favourable outcome. Eur J Cancer 1995; 31A:435-443.

8 Suzuki T, Bogenmann E, Shimada H, Stram D, Seeger RC. Lack of high-affinity nerve growth factor receptors in aggressive neuroblastomas. J Natl Cancer Inst 1993; 85:377-384.

9 Svendsen CN, ter Borg MG, Armstrong RJ, et al. A new method for the rapid and long term growth of human neural precursor cells. J Neurosci Methods 1998; 85:141-152.

10 Zorick TS, Lemke G. Schwann cell differentiation. Curr Opin Cell Biol 1996; 8:870-876.

11 Schodin BA, Tsomides TJ, Kranz DM. Correlation between the number of $\mathrm{T}$ cell receptors required for $\mathrm{T}$ cell activation and TCR-ligand affinity. Immunity 1996; 5:137-146.

12 Lavenius E, Gestblom C, Johansson I, Nanberg E, Pahlman $\mathrm{S}$. Transfection of TRK-A into human neuroblastoma cells restores their ability to differentiate in response to nerve growth factor. Cell Growth Differ 1995; 6:727-736.

13 Matsushima H, Bogenmann E. Expression of trkA cDNA in neuroblastomas mediates differentiation in vitro and in vivo. Mol Cell Biol 1993; 13:7447-7456.

14 Poluha W, Poluha DK, Ross AH. TrkA neurogenic receptor regulates differentiation of neuroblastoma cells. Oncogene 1995; 10:185-189.

15 Bromley SK, Burack WR, Johnson KG, et al. The immunological synapse. Annu Rev Immunol 2001; 19:375-396.

16 Han ZT, Zhu XX, Yang RY, et al. Effect of intravenous infusions of 12-O-tetradecanoylphorbol-13-acetate (TPA) in patients with myelocytic leukemia: preliminary studies on therapeutic efficacy and toxicity. Proc Natl Acad Sci USA 1998; 95:5357-5361.

17 Strair RK, Schaar D, Goodell L, et al. Administration of a phorbol ester to patients with hematological malignancies: preliminary results from a phase I clinical trial of 12-O-tetradecanoylphorbol-13-acetate. Clin Cancer Res 2002; 8:2512-2518.

18 Matthay KK. Intensification of therapy using hematopoietic stem-cell support for high-risk neuroblastoma. Pediatr Transplant 1999; 3(Suppl 1):72-77.

19 Tonini GP, Pistoia V. Molecularly guided therapy of neuroblastoma: a review of different approaches. Curr Pharm Des 2006; 12:2303-2317. 
20 Storch A, Paul G, Csete M, et al. Long-term proliferation and dopaminergic differentiation of human mesencephalic neural precursor cells. Exp Neurol 2001; 170:317-325.

21 Lavenius E, Parrow V, Nanberg E, Pahlman S. Basic FGF and IGF-I promote differentiation of human SH-SY5Y neuroblastoma cells in culture. Growth Factors 1994; 10:29-39.

22 Barzilay J, Kushtai G, Plaksin D, Feldman M, Eisenbach L. Expression of major histocompatibility class I genes in differentiating leukemic cells is temporally related to activation of c-fos proto-oncogene. Leukemia 1987; 1:198-204.

23 Bernard DJ, Courjal F, Maurizis JC, et al. Effect of epidermal growth factor in HLA class I and class II transcription and protein expression in human breast adenocarcinoma cell lines. $\mathrm{Br}$ J Cancer 1992; 66:88-92.

24 la Sala A, Corinti S, Federici M, Saragovi HU, Girolomoni G. Ligand activation of nerve growth factor receptor TrkA protects monocytes from apoptosis. J Leukoc Biol 2000; 68:104-110.

25 Leon JA, Gutierrez MC, Jiang H, et al. Modulation of the antigenic phenotype of human breast carcinoma cells by modifiers of protein kinase $\mathrm{C}$ activity and recombinant human interferons. Cancer Immunol Immunother 1992; 35:315-324.

26 Bassi V, De Riu S, Feliciello A, et al. Intercellular adhesion molecule-1 is upregulated via the protein kinase $\mathrm{C}$ pathway in human thyroid carcinoma cell lines. Thyroid 1998; 8:23-28.

27 Hunakova L, Sedlak J, Klobusicka M, Sulikova M, Chorvath B. Phorbol ester (TPA)-induced differential modulation of cell surface antigens in human pluripotential leukemia (K-562) cell line: effects of protein kinase inhibitors with broad- and PKC selective inhibitory activity. Neoplasma 1995; 42:249-253.

28 Raychaudhuri SK, Raychaudhuri SP, Weltman H, Farber EM. Effect of nerve growth factor on endothelial cell biology: proliferation and adherence molecule expression on human dermal microvascular endothelial cells. Arch Dermatol Res 2001; 293:291-295.

29 Vertuani S, Dubrovska E, Levitsky V, et al. Retinoic acid elicits cytostatic, cytotoxic and immunomodulatory effects on uveal melanoma cells. Cancer Immunol Immunother 2007; 56:193-204.

30 Barber DF, Faure M, Long EO. LFA-1 contributes an early signal for NK cell cytotoxicity. J Immunol 2004; 173:3653-3659.

31 Rouas-Freiss N, Moreau P, Menier C, Carosella ED. HLA-G in cancer: a way to turn off the immune system. Semin Cancer Biol 2003; 13:325-336.

32 Gunturi A, Berg RE, Forman J. The role of CD94/NKG2 in innate and adaptive immunity. Immunol Res 2004; 30:29-34.

33 Bamberger AM, Jenatschke S, Schulte HM, Loning T, Bamberger MC. Leukemia inhibitory factor (LIF) stimulates the human HLA-G promoter in JEG3 choriocarcinoma cells. $J$ Clin Endocrinol Metab 2000; 85:3932-3936.

34 Shido F, Ito T, Nomura S, et al. Endoplasmic reticulum aminopeptidase-1 mediates leukemia inhibitory factor-induced cell surface human leukocyte antigen-G expression in JEG-3 choriocarcinoma cells. Endocrinology 2006; 147:1780-1788.

35 Llano M, Lee N, Navarro F, et al. HLA-E-bound peptides influence recognition by inhibitory and triggering CD94/NKG2 receptors: preferential response to an HLA-G-derived nonamer. Eur J Immunol 1998; 28:2854-2863.

36 Whitelegg A, Barber LD. The structural basis of T-cell allorecognition. Tissue Antigens 2004; 63:101-108.

37 Obin M, Mesco E, Gong X, et al. Neurite outgrowth in PC12 cells. Distinguishing the roles of ubiquitylation and ubiquitindependent proteolysis. J Biol Chem 1999; 274:11789-11795.

38 Warzynski MJ, Graham DM, Axtell RA, Higgins JV, Hammers YA. Flow cytometric immunophenotyping test for staging/monitoring neuroblastoma patients. Cytometry 2002; 50:298-304.

39 Torsteinsdottir S, Masucci MG, Ehlin-Henriksson B, et al. Differentiation-dependent sensitivity of human B-cell-derived lines to major histocompatibility complex-restricted T-cell cytotoxicity. Proc Natl Acad Sci USA 1986; 83:5620-5624.

(Supplementary information is available at Cell Research's website.) 